\title{
Multimodal non-rigid registration methods based on local variability measures in computed tomography and magnetic resonance brain images
}

\author{
Isnardo Reducindo', Aldo R. Mejia-Rodriguez²,3, Edgar R. Arce-Santana1, Daniel U. Campos- \\ Delgado', Flavio Vigueras-Gomez' ${ }^{1}$ Elisa Scalco ${ }^{3}$, Anna M. Bianchi², Giovanni M. Cattaneo ${ }^{4}$, \\ Giovanna Rizzo 3 \\ ${ }^{1}$ Faculty of Sciences, Autonomous University of San Luis Potosi, Mexico \\ ${ }^{2}$ Bioengineering Department, Politecnico di Milano, Milan, Italy \\ ${ }^{3}$ Institute of Molecular Bioimaging and Physiology (IBFM), Italian National Research Council (CNR), Milan, Italy \\ ${ }^{4}$ Medical Physics Department, Ospedale San Raffaele, Scientific Institute, Milan, Italy \\ E-mail: ducd@fciencias.uaslp.mx
}

Received on 5th April 2013

Revised on 29th January 2014

Accepted on 23rd February 2014

\section{Introduction}

Image registration (IR) is an important research topic addressed through image processing and optimisation tools. IR can be divided into two main streams: rigid or parametric registration, and elastic or non-rigid registration (NRR) $[1,2]$. The parametric registration has been studied extensively [3], where techniques based on gradient descent $[4,5]$ are most commonly used to optimise a similarity measure (e.g. mutual information $[6-8,34]$ ) and to obtain the spatial transformation (e.g. affine or perspective [9]) that aligns the target and source images [1,2]. Other options based on global optimisation methods such as genetic algorithms [10] and particle filtering (PF) [11], have begun to play an important role in this field $[12,13]$. In fact, NRR is a more complex and involved problem, especially for multimodal images, where the selected images could not have an intensity correspondence between them, because of the acquisition with different imaging technologies or acquisition parameters. Thus, direct NRR techniques based on intensity alignment cannot be employed. Nowadays, multimodal NRR has a vast number of applications in medical imaging [14]. For instance, multimodal NRR is useful in radiotherapy (RT), where different types of medical images could lead to better identifying the structures of interest for the RT plan, such as the tumour and the organs at risk, and for studying the efficiency of RT and its collateral effects $[14,15]$. For example, when three-dimensional (3D) conformal radiotherapy treatment planning is employed for tumour treatment, the relative position between the tumour and its adjacent tissues could be obtained accurately through analysing the medical datasets, which fuse the information of functional and anatomical images [15]. In the literature, the most common methods to solve the elastic registration employ splines, where a family of functions is used to approximate the complex deformations, but the main drawback of these methods is their complexity and high computational cost

[16-18], mostly in terms of the number of interpolation points.

As described in [19], a most recent proposal to solve the NRR problem is based on iterative optical flow (OF) in order to find the deformation vector field that aligns the target and source images, after conducting an initial parametric registration by using the $\mathrm{PF}$. This combined algorithm $(\mathrm{PF}+$ OF) has shown promising results in $[20$, 
21]. Nonetheless, an important restriction of this approach is the hypothesis of intensity constancy between the target and source images, restricting the methodology to monomodal images. In this context, this work pursues to overcome the monomodal restriction of the PF + OF algorithm in [19], by applying a local intensity mapping over the images, after an initial parametric registration. In this proposal, the multimodal images are mapped into a space where their intensities can be compared, in order to perform an OF algorithm iteratively, as proposed in [22].

The rest of the paper is organised as follows: in Section 2.1, we briefly describe the monomodal NRR based on PF + OF, and in Section 2.2 the proposed multimodal NRR methodology is detailed; Section 2.3 presents the characteristics of the images datasets (clinical and synthetic) to perform the evaluation, and in Section 2.4 the standard indices used to evaluate the accuracy of the NRR are described. Section 3 presents the NRR results for the synthetic and clinical datasets, and in Section 4, we discuss those findings. Finally, in Section 5, the principal conclusions about the algorithm performance in multimodal NRR of anatomical structures of interest in RT are outlined.

\section{Methodology}

The NRR problem can be formulated as to find the displacements vector field $V(\boldsymbol{r})$ such that it can align a source image $\left(I_{\mathrm{S}}\right)$, with a target one $\left(I_{\mathrm{T}}\right)$. Then, the problem can be mathematically written as follows

$$
I_{\mathrm{T}}(\boldsymbol{r})=F\left[I_{\mathrm{S}}(\boldsymbol{r}+V(\boldsymbol{r}))\right]
$$

where $\boldsymbol{r}=(x, y)^{\top}$ denotes a position within the rectangular domain $\Omega \subset \mathbb{R}^{2}$ of the images, and $F[\cdot]$ represents the relation between the intensities of both images $I_{\mathrm{T}}$ and $I_{\mathrm{S}}$. According to (1), $F$ is the identity if the two images are monomodal, and the registration problem can be formulated as to find the OF between them. However, standard OF techniques can only find the correspondence between small displacements in the pixels of the images $\left(I_{\mathrm{T}}, I_{\mathrm{S}}\right)$, and then it is recommended to have an initial approximation $d_{0}(\boldsymbol{r})$ of the vector field $V(\boldsymbol{r})$ by using a parametric registration algorithm. Thus, the NRR problem is addressed in two steps: first an initial parametric registration is performed, and next an iterative OF solution is computed [19]. This approach is described briefly in the following subsection.

\subsection{Monomodal NRR based on PF + OF}

The basic idea of the parametric registration based on PF, proposed in [13], is to estimate the parameter vector $\boldsymbol{\theta}$ of a geometrical transformation $T(\boldsymbol{r} \mid \boldsymbol{\theta})$ (e.g. affine or perspective) applied to each pixel $\boldsymbol{r}$ by a stochastic search over an optimisation surface (cost function) [23], where the estimated parameter vector $\hat{\boldsymbol{\theta}}$ is a statistic over the particles (e.g. the mean value). For specific details about the implementation of the parametric registration based on PF, the reader refers to $[11,13,21,24]$. Once the parametric registration has been carried out, an initial estimation of the elastic vector field $d_{0}(\boldsymbol{r})=\boldsymbol{r}-T(\boldsymbol{r} \hat{\boldsymbol{\theta}})$ can be obtained, and the remaining displacements $d_{i}(\boldsymbol{r})$ that accomplish the NRR between the target $I_{\mathrm{T}}$ and the transformed $\tilde{I}_{\mathrm{S}}(\boldsymbol{r}) \triangleq I_{\mathrm{S}}\left(\boldsymbol{r}+d_{0}(\boldsymbol{r})\right)$ images are expected to be small. If the images are monomodal, it is possible to find these displacements by using an OF technique without parameterising the deformation space. Our approach for OF estimation is based on optimising the following quadratic energy function

$$
\begin{aligned}
\Psi(d(\boldsymbol{r}))= & \sum_{\forall \boldsymbol{r}}\left[I_{\mathrm{T}}(\boldsymbol{r})-\tilde{I}_{\mathrm{S}}(\boldsymbol{r}+d(\boldsymbol{r}))\right]^{2} \\
& +\lambda \sum_{\langle\boldsymbol{r}, \boldsymbol{s}\rangle}\|d(\boldsymbol{r})-d(\boldsymbol{s})\|_{2}^{2}
\end{aligned}
$$

where $\langle\boldsymbol{r}, \boldsymbol{s}\rangle$ denotes that pixels $\boldsymbol{r}$ and $\boldsymbol{s}$ are neighbours, $\lambda$ is a regularisation term to control flow homogeneity and $\|\cdot\|_{2}$ represents the Euclidean norm. Note that (2) describes a discrete version of the classical Horn-Schunck method [25], which is the basis of most OF algorithms proposed in the literature $[26,35]$. To optimise the non-linear quadratic cost function in (2), the data term can be linearised by conducting a first-order Taylor approximation

$$
\tilde{I}_{\mathrm{S}}(\boldsymbol{r}+d(\boldsymbol{r})) \simeq \tilde{I}_{\mathrm{S}}(\boldsymbol{r})+\nabla \tilde{I}_{\mathrm{S}}(\boldsymbol{r})^{\top} d(\boldsymbol{r})
$$

where $\nabla \tilde{I}_{\mathrm{S}}(\boldsymbol{r})$ is the gradient of $\tilde{I}_{\mathrm{S}}$ and $(\cdot)^{\top}$ denotes transposition. Therefore the minimum solution for the quadratic problem in (2) can be obtained by a direct calculation of the stationary optimality conditions with respect to $d(\boldsymbol{r})$, and then solving the resulting system of linear equations with efficient iterative methods (e.g. Gauss-Seidel technique [27]). For more implementation details of this strategy, the reader is referred to $[19,20]$.

Hence, the initial estimation $d_{0}(\boldsymbol{r})$ can be refined by accumulating the displacements obtained after solving the optimisation in (2) recursively. Thus, the monomodal PF + $\mathrm{OF}$ algorithm can be summarised in the next three steps:

1. Parametric registration: Find the parameters vector $\hat{\boldsymbol{\theta}}$ of the perspective transformation $T(\boldsymbol{r} \mid \hat{\boldsymbol{\theta}})$ that provides the best alignment between $I_{\mathrm{T}}(\boldsymbol{r})$ and $I_{\mathrm{S}}(\boldsymbol{r})$, and compute the initial (rigid) displacement vector field $d_{0}(\boldsymbol{r})$. In this work, the parameters vector is defined as $\hat{\boldsymbol{\theta}}=\left[d_{x}, d_{y}, e_{x}, e_{y}, s_{x}, s_{y}\right]^{\top}$ where $\phi$ denotes the rotation (degrees), $d_{x}$ and $d_{y}$ the displacements in $x$ - and $y$-axis $(\mathrm{mm}), e_{x}$ and $e_{y}$ are scales, and $s_{x}$ and $s_{y}$ are skewness in each axis.

2. $O F$ : Find the displacements between $I_{\mathrm{T}}$ and $\tilde{I}_{\mathrm{S}}$ by an iterative scheme of OF, $d(\boldsymbol{r})=d_{1}(\boldsymbol{r})+d_{2}(\boldsymbol{r})+\cdots+d_{k}(\boldsymbol{r})$, until convergence is achieved; where at each $k$ th iteration the $\mathrm{OF}$ is computed over $I_{\mathrm{T}}(\boldsymbol{r})$ and $\tilde{I}_{\mathrm{S}}^{k}(\boldsymbol{r})$, where $\tilde{I}_{\mathrm{S}}^{k}(\boldsymbol{r}) \triangleq \tilde{I}_{\mathrm{S}}\left(\mathbf{r}+\sum_{i=1}^{k-1} d_{i}(\boldsymbol{r})\right)$.

3. NRR: Finally, we can obtain the vector field of the non-rigid deformation by adding the rigid vector field to the one obtained by the iterative OF, that is, $V(\boldsymbol{r}) \triangleq d_{0}(\boldsymbol{r})+d(\boldsymbol{r})$. Consequently, we can deduce the elastic registered image as $I_{\mathrm{R}}(\boldsymbol{r}) \triangleq I_{\mathrm{S}}(\boldsymbol{r}+V(\boldsymbol{r}))$.

\subsection{Multimodal algorithm for NRR based on local variability measures}

In the case of multimodal NRR, the algorithm in [19] suggests approximating the transfer function of intensities between the target and source images by using the joint histogram, but this approach is only feasible if the intensity relation $F[\cdot]$ in (1) is injective. Hence, in this work, we propose an extension of this methodology in order to overcome these limitations by applying an intensity mapping over the images, once the target and source images are roughly aligned by a 
parametric registration, by using measures that describe the intensity variability around each pixel [22]. This mapping transforms both images into a space where each pixel intensity in one image could be compared with its corresponding in the other image, despite their intrinsic multimodal characteristics; namely, establishing a mapping $G[\cdot]$ such that

$$
G\left[I_{\mathrm{T}}(\boldsymbol{r})\right]=G\left[I_{\mathrm{S}}(\boldsymbol{r}+V(\boldsymbol{r}))\right]
$$

In order to define this mapping, we propose to employ measures that do not depend on the grey level of the pixels, but on their intensity variability around neighbour elements. Two measures that meet the above description are the entropy and the variance, computed over a window centred in the pixel of interest, which we call local variability measures (LVM). In addition, we also propose to employ a combination of these two metrics in order to take advantage of the local information that they capture. Euclidean and maximum weights are used, similarly to the two and infinity norms of two-dimensional (2D) vectors. The proposed methodology $\mathrm{PF}+\mathrm{LVM}+\mathrm{OF}$ for multimodal NRR adds two intermediate steps to the algorithm described in the previous subsection (see flowchart of the proposed method in Fig. 1):

\section{Parametric registration.}

2. Intensity mapping based on LVM. Apply the mapping $G[\cdot]$ based on a LVM over all the pixels rin the images $I_{\mathrm{T}}(\boldsymbol{r})$ and $\tilde{I}_{\mathrm{S}}(\boldsymbol{r})$. That is, compute the intensity mapping, and obtain $\tilde{I}_{\mathrm{T}}(\boldsymbol{r}) \triangleq G\left[I_{\mathrm{T}}(\boldsymbol{r})\right]$ and $\tilde{I}_{\mathrm{S}}^{0}(\boldsymbol{r}) \triangleq G\left[I_{\mathrm{S}}\left(\boldsymbol{r}+d_{0}(\boldsymbol{r})\right)\right]$, according to the following four proposals

$$
\begin{gathered}
G_{1}[I(\boldsymbol{r})]=\sum_{\boldsymbol{s} \in N_{\boldsymbol{r}}} p_{\boldsymbol{r}}(I(\boldsymbol{s})) \log [I(\boldsymbol{s})] \\
G_{2}[I(\boldsymbol{r})]=\sum_{\boldsymbol{s} \in N_{\boldsymbol{r}}} p_{\boldsymbol{r}}(I(\boldsymbol{s}))\left[\mu_{\boldsymbol{r}}-I(\boldsymbol{s})\right]^{2} \\
G_{3}[I(\boldsymbol{r})]=\sqrt{G_{1}[I(\boldsymbol{r})]^{2}+G_{2}[I(\boldsymbol{r})]^{2}} \\
G_{4}[I(\boldsymbol{r})]=\max _{r}\left\{G_{1}[I(\boldsymbol{r})], G_{2}[I(\boldsymbol{r})]\right\}
\end{gathered}
$$

where $G_{1}$ represents the LVM using 'entropy', $G_{2}$ using 'variance', $G_{3}$ is an Euclidean weight between variance and entropy, and $G_{4}$ is selecting the maximum value between variance and entropy at each pixel. In addition, $N_{r}$ represents the set of pixels of an $n \times n$ window centred at $r$, $p_{r}(I(s))$ is the local probability distribution of the image intensities $I(s)$ within $N_{r}$ and $\mu_{r}$ is the average value of the intensity $I(s)$ with $s \in N_{r}$. In this work, the size of $N_{r}$ is $7 \times$ 7 pixels, as suggested in [22].
3. Equalisation: After the transformation, the intensities of the images $\left(\tilde{I}_{\mathrm{T}}\right.$ and $\tilde{I}_{\mathrm{S}}^{0}$ ) could have small values and could be concentrated in a short dynamic range. For this reason, it is necessary to rescale the two-image intensities and to apply a histogram equalisation [9].

4. $O F$ : Find the remaining displacements between $I_{\mathrm{T}}$ and $\tilde{I}_{\mathrm{S}}^{0}$ by an OF iterative scheme.

5. Non-rigid registration.

\subsection{Clinical and synthetic image datasets}

Computed tomography (CT) and magnetic resonance (MR) clinical studies of three patients with cerebral tumour were selected for evaluation purposes. In these studies, it is possible to observe deformations of the brain structures in the axial plane, because of tumour structures (primary tumour and metastatic or secondary tumours) shrinkage as a result of the medical treatment. These patients underwent pre-operative diagnostic and RT treatment at the San Raffaele Hospital in Milan, Italy. The dataset was composed by CT scans and MR T1 weighted images before the RT treatment (CT-pre and MR-pre), and MR T1 weighted images after the treatment (MR-post). Owing to the effectiveness of the RT treatment in all clinical cases, some tumours were no longer visible after the medical intervention, and to avoid information inconsistencies in the registration process, we decided to use $2 \mathrm{D}$ images instead of the whole 3D scan. Thus, from the three clinical studies, ten pairs of CT/MR 2D images from different sections of the head were carefully selected, in order to have anatomic correspondence between pre- and post-images, and to study different morphological structures. Each image has a dimension of $512 \times 512$ pixels with a pixel size of $1.0 \times 1.0$ $\mathrm{mm}$. The slice-by-slice correspondence between CT-pre and both MR images (pre and post) was obtained by using the software available in the Eclipse Treatment Planning System [28].

In addition to the clinical dataset, we generated new synthetic data for the algorithm evaluation, where two MR-pre images of different sections of the head were deformed in a controlled way (three deformations per image) by using the moving least squares algorithm [29], in order to simulate a tumour evolution during RT treatment. In this way, the NRR process was evaluated between a CT-pre image and the synthetically modified MR-pre image. Moreover, our interest was to use the proposed algorithm to follow the tumour evolution during RT treatment. For this reason, in the clinical images, the proposed algorithm was adapted to compute the OF over the tumour area and its near structures, where the displacements at the edges of the selected regions were controlled through the regularisation term in (2), and by the vector field obtained by PF.

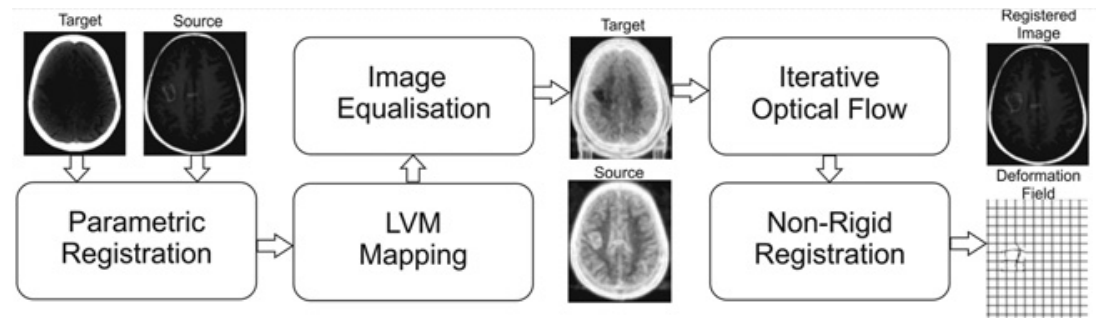

Fig. 1 Flowchart of the proposed algorithm for multimodal NRR based on LVM and OF 


\subsection{Registration accuracy}

First, an expert physician evaluated the results of the registration process by visual inspection, in order to label the performance as acceptable or not acceptable from a clinical point of view. Then, segmentations of the structures of interest (brain cortex and tumour in synthetic dataset, ventricles and tumour in clinical dataset) were carried out for each of the four images obtained after the registration with the PF + LVM + OF method, and for the original PF + OF implementation (without LVM). For analysis purposes, we define $\mathrm{LVM}_{k}$ as the PF + LVM + OF approach by using the mapping $G_{k}$, as described in (5)-(8), and No-LVM for just $\mathrm{PF}+\mathrm{OF}$. Owing to the difficulty in properly locating anatomical structures just by using the CT image, a CT-pre/ MR-pre correspondence was used to ensure a proper segmentation of the structures of interest in the CT images, where contour delineations of the structures in the MR-pre images were used for this purpose. These segmentations were performed by an expert observer through the MIPAV software [30] (see Fig. 2). From these segmentations, differences in the anatomical structures before and after the registration process were calculated by using standard indices usually adopted for accuracy assessment: the area difference (ADiff), the dice similarity coefficient (DSC), the average symmetric distance (ASD) and the maximum symmetric distance (DMax) [20, 31-33].

First, ADiff and DSC are indices that measure the overlap between two structures described by binary masks. Hence, given two different binary masks $A$ and $B$, representing two sets of pixels delimited by a contour, the index ADiff can be defined as

$$
\mathrm{ADiff}=|(A \cup B) \backslash(A \cap B)|=|(A \backslash B) \cup(B \backslash A)|
$$

and the DSC index

$$
\mathrm{DSC}=2 \frac{|A \cap B|}{|A|+|B|}
$$

where $|\cdot|$ denotes the cardinality of the set, $\backslash$ represents the set difference, and $\cup$ and $\cap$ are the union and intersection, respectively. In our evaluations, we had an equivalence of the area associated with each pixel in the images. In this way, it is possible to quantify ADiff in area units $\left(\mathrm{mm}^{2}\right)$. Note that ADiff equals to zero means a complete overlap. On the other hand, DSC ranges from 0 (no spatial overlap) to 1 (complete overlap).
Likewise, given contours $C_{A}$ and $C_{B}$ of the binary masks $A$ and $B$ respectively, the ASD index calculates the average distance between two contours. To compute it, first calculates for each contour pixel $\boldsymbol{p}_{B} \in C_{B}$, the Euclidean distance between $\boldsymbol{p}_{B}$ and its closest pixel in $C_{A}$. In order to provide symmetry, the same process is applied to the contour pixels of $\boldsymbol{p}_{A} \in C_{A}$ with respect to $C_{B}$. The ASD is then defined as the average of all computed distances

$$
\operatorname{ASD}=\frac{1}{\left|C_{A}\right|+\left|C_{B}\right|}\left(\sum_{\boldsymbol{p}_{A} \in C_{A}} d\left(\boldsymbol{p}_{A}, C_{B}\right)+\sum_{\boldsymbol{p}_{B} \in C_{B}} d\left(\boldsymbol{p}_{B}, C_{A}\right)\right)
$$

where $d(\boldsymbol{p}, C)=\min _{\boldsymbol{q} \in C}\|\boldsymbol{p}-\boldsymbol{q}\|_{2}$. Therefore a perfect match between contours $C_{A}$ and $C_{B}$ is expressed as a zero value for ASD. Meanwhile, DMax calculates the maximum distance between the analysed overlapped contours, where zero means a perfect overlap. As a result, DMax provides an estimation of the worst local distance mismatch and is defined as

$$
\operatorname{DMax}=\max \left\{\max _{\boldsymbol{p}_{A} \in C_{A}} d\left(\boldsymbol{p}_{A}, C_{B}\right), \max _{\boldsymbol{p}_{B} \in C_{B}} d\left(\boldsymbol{p}_{B}, C_{A}\right)\right\}
$$

To avoid bias in the quantitative evaluation owing to differences in the dimensions of the structures of interest, the resulting indices were analysed for each type of anatomical structure (brain cortex, tumour and ventricles). So the evaluation results are presented as mean \pm std for each structure. Additionally, in the synthetic images, eight markers were placed around the tumour with the goal of estimating the Euclidean distance between the coordinates of the markers in the images, before and after the registration process.

\section{Results}

\subsection{Synthetic images}

Fig. 3 shows an example of the registration results obtained by the different LVM proposed in this paper, where a CT-pre image is used as $I_{\mathrm{T}}$, and an MR-pre synthetically deformed as $I_{\mathrm{S}}$. In the parametric registration, the $\mathrm{PF}$ estimated the following parameters $\hat{\boldsymbol{\theta}}=[-0.838$, $-4.805,-4.218,1.074,1.066,-0.020,-0.022]^{\top}$. Table 1 presents the overall results for the brain contour and tumour by using the accuracy indices described earlier. The results

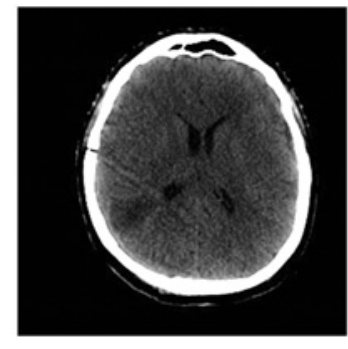

$a$

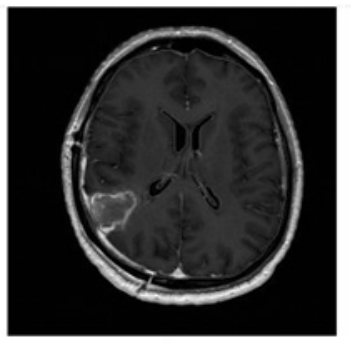

b

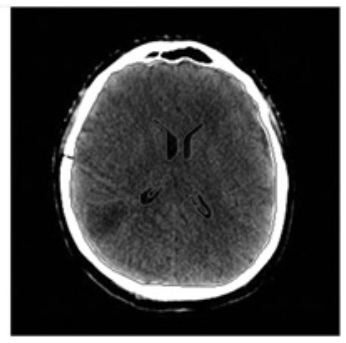

c

Fig. 2 Example of the set of images used for the algorithm evaluation

$a$ CT-pre with contours of the anatomical structures of interest

$b$ MR-pre with contours of the anatomical structures of interest

$c$ CT-pre with contours of the anatomical structures of interest obtained from MR-pre 


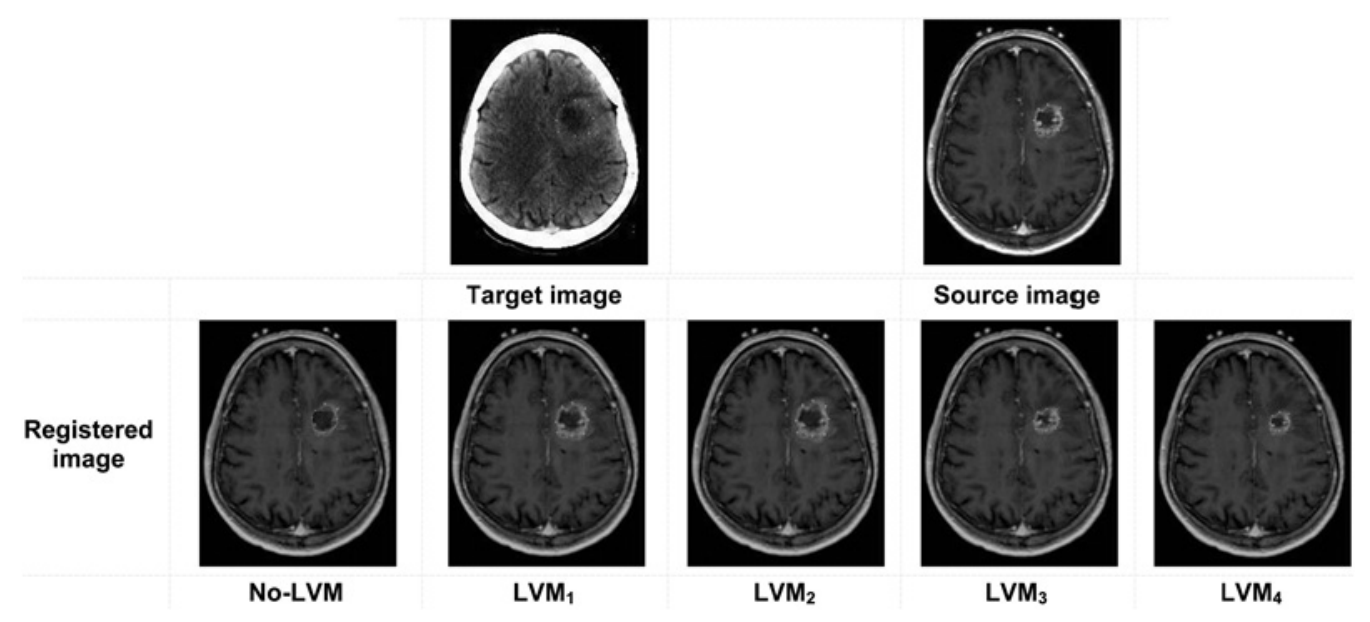

Fig. 3 Example of NRR results of the synthetic dataset for the four LVM and original PF + OF algorithm (No-LVM)

The first row shows the CT-pre and MR-pre synthetically deformed, target and source respectively, with markers in the structures of interest. The second row shows the registration result by using the algorithm ( $\mathrm{PF}+\mathrm{OF}$ ), without LVM mapping (first column) and with each LVM mapping (columns two to five)

show a similarity in ADiff for the brain contour, before and after the registration process (for any LVM); while in the tumour, a considerable decrease of about $60 \%$ is observed in $\mathrm{LVM}_{1}$ and $\mathrm{LVM}_{4}$, and an increase of about $50 \%$ is presented for $\mathrm{LVM}_{2}, \mathrm{LVM}_{3}$, and No-LVM. For the ASD index, the NRR results for the brain structure show a slight decrease only by $\mathrm{LVM}_{1}$ with respect to the initial registration value. At the same time, an increment of about $20 \%$ is found in ASD for the rest of the LVM. In the tumour, a considerable decrease (around 45\%) was accomplished by $\mathrm{LVM}_{1}$ and $\mathrm{LVM}_{4}$, with respect to the ASD value before registration; $\mathrm{LVM}_{2}, \mathrm{LVM}_{3}$ and No-LVM presented an increment of approximately twice its initial value. Analysing the brain contour for the DMax index, a slightly decrease is observed in $\mathrm{LVM}_{1}$ and $\mathrm{LVM}_{4}$ (around $20 \%$ ) although $\mathrm{LVM}_{2}$ and $\mathrm{LVM}_{3}$ remain approximately at the same value before the NRR process, and No-LVM presented an increase of $23 \%$. For the tumour, a $20 \%$ decrease is presented in $\mathrm{LVM}_{1}$ and $\mathrm{LVM}_{4}$ for DMax. Although a $35 \%$ increase is presented with the other two LVM and No-LVM case. Finally, the NRR average performance for the brain structure with the DSC index was always above 0.9 for all cases, before and after the NRR process. In the tumour, DSC shows an increase after the NRR process with $\mathrm{LVM}_{1}(10 \%)$ and $\mathrm{LVM}_{4}(7 \%)$, and a decrease of about $15 \%$ with $\mathrm{LVM}_{2}, \mathrm{LVM}_{3}$ and No-LVM.
The overall evaluation in Table 1 shows that the NRR with $\mathrm{LVM}_{1}$ achieved the best mean index in $75 \%$ of the cases, and the lowest variability (std) in $63 \%$.

In addition to the accuracy indices, the Euclidean error is calculated between the tumour markers before and after the NRR process in Table 2. For each deformation (Def), the mean \pm std value of the distance between the eight markers around the tumour is presented for each LVM. In this table, we can observe a decrease in the mean error and its variability (std) for $\mathrm{LVM}_{1}$ and $\mathrm{LVM}_{4}(25 \%)$. Meanwhile, the error increased for $\mathrm{LVM}_{2}, \mathrm{LVM}_{3}$ and No-LVM (roughly 50\%).

\subsection{Clinical images}

Fig. 4 shows an example of the NRR process of the proposed approach with clinical data, where it is possible to visually assess the performance of the registration for each LVM. The CT-pre and MR-post images $\left(I_{\mathrm{T}}\right.$ and $I_{\mathrm{S}}$, respectively) show the tumour before and after the RT treatment, located in the centre of the left hemisphere (Fig. 4 first column, rows one and two). Therefore the visual inspection consisted in evaluating whether the registration process was able to recover the deformation (shrinking) suffered by the tumour owing to the RT treatment. Taking this information into account, $\mathrm{LVM}_{1}, \mathrm{LVM}_{3}$ and $\mathrm{LVM}_{4}$ have an acceptable

Table 1 Mean \pm standard deviation of ADiff (area difference), ASD (average symmetric distance), DMax (maximum symmetric distance) and DSC (dice similarity coefficient) obtained before the registration process, with the four LVM (entropy, variance, and Euclidean and maximum weights between them) and with the No-LVM version, for the three anatomical structures of interest in the synthetic images of Fig. 3

\begin{tabular}{|c|c|c|c|c|c|c|c|}
\hline \multirow[t]{2}{*}{ Index } & \multirow[t]{2}{*}{ Anatomical structure } & \multicolumn{6}{|c|}{ Mean \pm Std. Dev. } \\
\hline & & Before registration & No-LVM & $\mathrm{LVM}_{1}$ & $\mathrm{LVM}_{2}$ & $\mathrm{LVM}_{3}$ & $\mathrm{LVM}_{4}$ \\
\hline ADiff, $\mathrm{mm}^{2}$ & $\begin{array}{l}\text { tumour } \\
\text { brain }\end{array}$ & $\begin{array}{l}0.283 \pm 0.049 \\
0.005 \pm 0.002\end{array}$ & $\begin{array}{l}0.328 \pm 0.094 \\
0.008 \pm 0.005\end{array}$ & $\begin{array}{l}0.100 \pm 0.073 \\
0.004 \pm 0.003\end{array}$ & $\begin{array}{l}0.445 \pm 0.187 \\
0.005 \pm 0.002\end{array}$ & $\begin{array}{l}0.432 \pm 0.132 \\
0.007 \pm 0.004\end{array}$ & $\begin{array}{l}0.145 \pm 0.096 \\
\mathbf{0 . 0 0 4} \pm \mathbf{0 . 0 0 2}\end{array}$ \\
\hline $\mathrm{ASD}, \mathrm{mm}$ & $\begin{array}{l}\text { tumour } \\
\text { brain }\end{array}$ & $\begin{array}{l}2.698 \pm 0.729 \\
0.340 \pm 0.075\end{array}$ & $\begin{array}{l}3.992 \pm 1.001 \\
0.542 \pm 0.185\end{array}$ & $\begin{array}{l}1.427 \pm 0.587 \\
0.331 \pm 0.086\end{array}$ & $\begin{array}{l}4.560 \pm 1.226 \\
0.466 \pm 0.178\end{array}$ & $\begin{array}{l}4.284 \pm 1.063 \\
0.495 \pm 0.170\end{array}$ & $\begin{array}{l}1.735 \pm 0.766 \\
0.415 \pm 0.205\end{array}$ \\
\hline DMax, mm & $\begin{array}{l}\text { tumour } \\
\text { brain }\end{array}$ & $\begin{array}{l}6.143 \pm 2.120 \\
3.549 \pm 0.992\end{array}$ & $\begin{array}{l}8.600 \pm 1.989 \\
4.368 \pm 0.992\end{array}$ & $\begin{array}{l}4.778 \pm 1.967 \\
2.730 \pm 0.846\end{array}$ & $\begin{array}{l}8.327 \pm 1.750 \\
3.413 \pm 1.312\end{array}$ & $\begin{array}{l}8.371 \pm 2.139 \\
3.276 \pm 0.518\end{array}$ & $\begin{array}{l}4.837 \pm 1.929 \\
2.867 \pm 0.449\end{array}$ \\
\hline DSC & $\begin{array}{l}\text { tumour } \\
\text { brain }\end{array}$ & $\begin{array}{l}0.817 \pm 0.042 \\
0.995 \pm 0.001\end{array}$ & $\begin{array}{l}0.729 \pm 0.051 \\
0.993 \pm 0.002\end{array}$ & $\begin{array}{l}0.906 \pm 0.036 \\
0.996 \pm 0.001\end{array}$ & $\begin{array}{l}\mathbf{0 . 6 6 7} \pm \mathbf{0 . 1 2 6} \\
0.994 \pm 0.002\end{array}$ & $\begin{array}{l}0.693 \pm 0.094 \\
0.993 \pm 0.003\end{array}$ & $\begin{array}{l}0.883 \pm 0.049 \\
0.995 \pm 0.002\end{array}$ \\
\hline
\end{tabular}

A bold font highlights the best results per row after NRR based on the mean values 
Table 2 Euclidean distance $(\mathrm{mm})$ of the tumour markers (mean \pm standard deviation), before and after registration for each synthetic deformation (Def) of Fig. 3

\begin{tabular}{|c|c|c|c|c|c|c|c|}
\hline \multicolumn{2}{|c|}{ Deformations } & \multicolumn{6}{|c|}{ Mean \pm Std. Dev., $\mathrm{mm}$} \\
\hline & & Before registration & No-LVM & $\mathrm{LVM}_{1}$ & $\mathrm{LVM}_{2}$ & $\mathrm{LVM}_{3}$ & $\mathrm{LVM}_{4}$ \\
\hline \multirow[t]{3}{*}{ Image1 } & Def1 & $3.695 \pm 0.910$ & $5.650 \pm 3.244$ & $1.714 \pm 0.461$ & $8.019 \pm 1.283$ & $6.074 \pm 1.767$ & $1.675 \pm 1.106$ \\
\hline & Def2 & $5.383 \pm 1.081$ & $6.898 \pm 2.778$ & $3.761 \pm 2.799$ & $7.522 \pm 2.488$ & $7.270 \pm 2.146$ & $3.385 \pm 1.86$ \\
\hline & Def3 & $4.437 \pm 1.103$ & $6.303 \pm 3.099$ & $2.061 \pm 1.196$ & $8.369 \pm 1.052$ & $6.919 \pm 1.466$ & $2.428 \pm 1.504$ \\
\hline \multirow[t]{3}{*}{ Image2 } & Def1 & $3.571 \pm 2.267$ & $5.978 \pm 2.648$ & $3.169 \pm 1.397$ & $5.540 \pm 3.104$ & $5.892 \pm 3.364$ & $3.530 \pm 1.214$ \\
\hline & Def2 & $3.468 \pm 2.228$ & $5.500 \pm 2.205$ & $3.206 \pm 2.384$ & $5.021 \pm 2.984$ & $5.142 \pm 3.012$ & $3.621 \pm 2.538$ \\
\hline & Def3 & $6.364 \pm 3.999$ & $8.476 \pm 4.315$ & $5.400 \pm 2.896$ & $8.141 \pm 5.516$ & $8.367 \pm 2.680$ & $5.676 \pm 2.896$ \\
\hline
\end{tabular}

A bold font highlights the best results per row after NRR based on the mean values

performance from a clinical point of view (see Fig. 4 columns two, four and five) whereas $\mathrm{LVM}_{2}$ and No-LVM have not, since they predict a change in opposite direction (see Fig. 4 columns one and three). During the parametric registration, the following parameters $\hat{\boldsymbol{\theta}}=[-1.925$, $-1.241,0.286,0.936,0.919,-0.009,-0.062]^{\top} \quad$ were estimated by the PF. Meanwhile, the final deformation field $V(\boldsymbol{r})$ over a rectangular grid is shown in the third row of Fig. 4 for each LVM.

The quantitative analysis is presented in Table 3, where for each performance index the mean \pm std for the group of anatomical structures of interest in RT (ventricles and tumour) is shown. We decided to exclude the brain contour, because this structure depends on the parametric registration (as the synthetic data shows), and in our clinical cases, this step does not have a big impact in the final registration performance. However, it is necessary to continue considering the parametric registration, since in many clinical cases this step could be very helpful, as has been described in the literature [1-3, 14]. As shown in Table 3, for the ventricles and the tumour, a more evident decrease was presented in all LVM and No-LVM cases. For the ventricles, a decrease was found with all four LVM, where $\mathrm{LVM}_{2}$ showed the largest improvement $(15 \%)$.

Finally, the DSC in the ventricles shows a slight increase after NRR (about 8\%) with all LVMs and No-LVM scenarios. Analysing the tumour in Table 3, $\mathrm{LVM}_{2}$ and

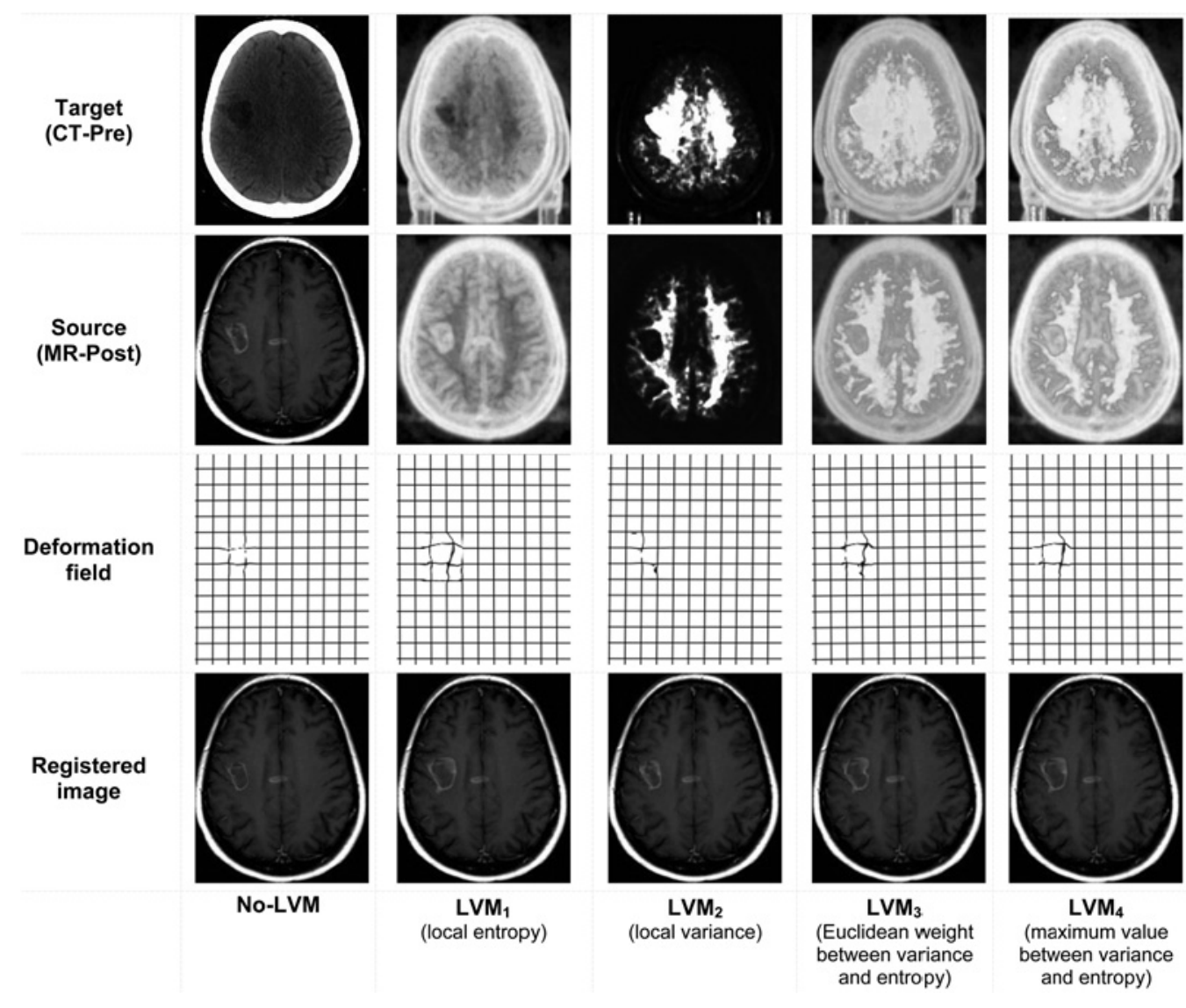

Fig. 4 Example of NRR results with clinical images using LVM and No-LVM version

The first row shows the CT-pre, used as a target, without LVM mapping (first column) and after applying the intesity mapping with each LVM (columns two to five); in the same way the MR-post, used as a source, is shown in the second row. The third row shows the obtained deformation field using the algorithm (PF + OF), without LVM mapping (first column) and with each LVM mapping (columns two to five). The fourth row shows the registration result, for No-LVM (first column) and for each LVM mapping (columns two to five) 
Table 3 Mean \pm standard deviation of ADiff (area difference), ASD (average symmetric distance), DMax (maximum symmetric distance) and DSC (dice similarity coefficient) obtained before the registration process, with the four LVM (entropy, variance, two-norm and infinity-norm) and with the No-LVM version for the three anatomical structures of interest in Fig. 4

\begin{tabular}{|c|c|c|c|c|c|c|c|}
\hline \multirow[t]{2}{*}{ Index } & \multirow[t]{2}{*}{ Anatomical structure } & \multicolumn{6}{|c|}{ Mean \pm Std. Dev. } \\
\hline & & Before registration & No-LVM & $\mathrm{LVM}_{1}$ & $\mathrm{LVM}_{2}$ & $\mathrm{LVM}_{3}$ & $\mathrm{LVM}_{4}$ \\
\hline ADiff, $\mathrm{mm}^{2}$ & $\begin{array}{l}\text { tumour } \\
\text { ventricles }\end{array}$ & $\begin{array}{l}0.420 \pm 0.263 \\
0.964 \pm 2.163\end{array}$ & $\begin{array}{l}0.389 \pm 0.350 \\
\mathbf{0 . 5 0 1} \pm \mathbf{0 . 8 7 9}\end{array}$ & $\begin{array}{l}0.252 \pm 0.365 \\
0.520 \pm 0.915\end{array}$ & $\begin{array}{l}0.415 \pm 0.292 \\
0.679 \pm 1.973\end{array}$ & $\begin{array}{l}0.366 \pm 0.310 \\
0.692 \pm 2.108\end{array}$ & $\begin{array}{l}0.278 \pm 0.340 \\
0.665 \pm 1.084\end{array}$ \\
\hline ASD, mm & $\begin{array}{l}\text { tumour } \\
\text { ventricles }\end{array}$ & $\begin{array}{l}4.265 \pm 3.285 \\
2.197 \pm 1.241\end{array}$ & $\begin{array}{l}4.804 \pm 4.265 \\
1.868 \pm 1.441\end{array}$ & $\begin{array}{l}3.517 \pm 4.123 \\
1.891 \pm 1.157\end{array}$ & $\begin{array}{l}4.669 \pm 3.752 \\
1.811 \pm 1.234\end{array}$ & $\begin{array}{l}4.009 \pm 3.926 \\
1.829 \pm 1.081\end{array}$ & $\begin{array}{l}3.590 \pm 3.694 \\
1.970 \pm 1.202\end{array}$ \\
\hline DMax, mm & $\begin{array}{l}\text { tumour } \\
\text { ventricles }\end{array}$ & $\begin{array}{l}9.398 \pm 4.046 \\
7.841 \pm 6.372\end{array}$ & $\begin{array}{c}10.362 \pm 5.713 \\
8.042 \pm 7.424\end{array}$ & $\begin{array}{l}7.980 \pm 4.710 \\
7.308 \pm 6.599\end{array}$ & $\begin{array}{c}10.144 \pm 4.843 \\
6.604 \pm 5.447\end{array}$ & $\begin{array}{l}9.031 \pm 5.228 \\
6.918 \pm 4.875\end{array}$ & $\begin{array}{l}8.368 \pm 4.560 \\
7.833 \pm 6.783\end{array}$ \\
\hline DSC & $\begin{array}{l}\text { tumour } \\
\text { ventricles }\end{array}$ & $\begin{array}{l}0.683 \pm 0.264 \\
0.608 \pm 0.198\end{array}$ & $\begin{array}{l}\mathbf{0 . 6 5 2} \pm \mathbf{0 . 3 2 2} \\
0.681 \pm 0.205\end{array}$ & $\begin{array}{l}0.746 \pm 0.319 \\
0.659 \pm 0.191\end{array}$ & $\begin{array}{l}0.661 \pm 0.289 \\
0.662 \pm 0.213\end{array}$ & $\begin{array}{l}0.710 \pm 0.301 \\
\mathbf{0 . 6 4 5} \pm \mathbf{0 . 2 1 4}\end{array}$ & $\begin{array}{l}0.741 \pm 0.294 \\
0.656 \pm 0.185\end{array}$ \\
\hline
\end{tabular}

A bold font highlights the best results per row after NRR based on the mean values

No-LVM did not improve ASD, DMax and DSC after the NRR process. An improvement in all four indices is found only for $\mathrm{LVM}_{1}, \mathrm{LVM}_{3}$ and $\mathrm{LVM}_{4}$; being the best improvements of $40 \%$ for the ADiff, $17 \%$ for the ASD, $15 \%$ for the DMax and $9 \%$ for the DSC, all of them by using $\mathrm{LVM}_{1}$.

\section{Discussion}

\subsection{Synthetic images}

In the analysis of the synthetic evaluation, only the brain contour, the biggest rigid structure presented in the images, and the tumour, which is the structure that presents complex deformations, were considered as structures of interest for RT. Therefore, as expected, the values of all four accuracy indices (ADiff, DSC, ASD and DMax) calculated for all the LVM and No-LVM at the contour of the brain did not change significantly compared to their values before NRR. For the tumour, an improvement was presented only for $\mathrm{LVM}_{1}$ and $\mathrm{LVM}_{4}$ in all indices, being $\mathrm{LVM}_{1}$ the one with the largest improvement. The analysis of the Euclidean error among markers in the tumour section provided an estimation of the NRR accuracy on specific points for a structure of interest; a new, the corresponding results show positive performance only for $\mathrm{LVM}_{1}$ and $\mathrm{LVM}_{4}$. However, by considering the results in [22], where LVM were first proposed for the registration of medical images, an acceptable performance was found in all four LVM. Moreover, the performance analysis in [22] was only based on the mean error of the entire vector field after applying the NRR algorithm; while in this paper, we analyse the performance of the different LVM methodologies by specifically addressing the anatomical structures of clinical interest.

\subsection{Clinical images}

The resulting images after the NRR process show that $\mathrm{LVM}_{1}$ (entropy) provides qualitatively and quantitatively the best results on the set of evaluation images, followed by $\mathrm{LVM}_{4}$ (maximum weight between entropy and variance), appropriately aligning the anatomical structures in MR-post with CT-pre. Although $\mathrm{LVM}_{2}$ and $\mathrm{LVM}_{3}$ (variance and Euclidean weight between both metrics) achieve an adequate alignment for some structures of interest, these metrics generated anatomically inconsistent deformations and changes in the textures of tissues in the registered images (figures omitted owing to space limitations). The inconsistent deformations obtained after NRR by $\mathrm{LVM}_{2}$ might be caused by highly textured (in MR) and low contrast areas (in CT), as in several brain regions, where the values obtained by computing local variance are constant because of low variability of the intensities in these regions. This phenomenon becomes similar to estimate the $\mathrm{OF}$ between two constant-intensity areas, where any displacement in any direction keeps unchanged the cost function. In the same way, within a homogeneous area, there is high probability of obtaining values of variance and entropy for two different locations with similar magnitudes, generating an identical problem for $\mathrm{LVM}_{3}$ (Euclidean weight). These effects explain the inconsistent deformations generated by $\mathrm{LVM}_{2}$ and $\mathrm{LVM}_{3}$, which do not occur in $\mathrm{LVM}_{1}$, because in highly textured areas, the entropy values are very different among pixels, owing to the high uncertainty in the intensities of these regions (see Fig. 4).

In fact, the quantitative analysis on the tumour shows that the best results for the NRR algorithm were obtained by using $\mathrm{LVM}_{1}$ and $\mathrm{LVM}_{4}$, despite some problems with the contrast in the CT-pre images. Moreover, the No-LVM approach does not provide good results to estimate the tumour deformation, because the tissue intensities at each modality (CT and MR) are very different. In contrast, the No-LVM approach gave the best results in terms of ADiff and DSC in the ventricles (along with $\mathrm{LVM}_{1}$ ) and also presented positive results for the ASD index (see Table 3 ). This behaviour could be related to the similarity of intensities in the ventricles, for both $\mathrm{CT}$ and MR.

The positive results found with the No-LVM implementation follow the performance shown in $[19,20]$, where it is stated that the use of the PF + OF method is a valuable tool for complex NRR problems. In the meantime, the negative results support the disadvantage of this methodology for multimodal cases, so that it reinforces the motivation for using LVM to achieve a multimodal registration that does not parameterise the elastic deformation space, in contrast to most of the methods reported in the literature [16-18]. Furthermore, a parameterisation of the deformation space (e.g. using splines) limits the vector fields that can be achieved and increases the computational cost, because the optimisation processes used by these methods are typically non-linear [16-18]. These two problems are solved with the technique proposed in this work $\mathrm{PF}+\mathrm{LVM}+\mathrm{OF}$, since each pixel can move independently, thus increasing the manifoldness of deformations that can be reproduced. Also, the $\mathrm{OF}$ 
estimation process is formulated as a quadratic optimisation over a convex surface [see (2)], and its global solution is computed by a system of linear equations, which ensures a low computational cost.

Making a comparison between the four LVM and the No-LVM implementation (see Tables 1-3), it is possible to see a consistent advantage of $\mathrm{LVM}_{1}$ (entropy) over the other LVM and No-LVM approach in mean performance, and even in some cases, with less variability. This idea suggests that the local entropy is the best performing LVM under a quantitative analysis of anatomical structures of interest for RT brain images. Furthermore, the results found in this work suggest that the PF $+\mathrm{LVM}+\mathrm{OF}$ algorithm could be useful in RT for some important tasks, such as the monitoring of medical treatment. Additionally, some aspects of the NRR methodology could be still improved in future studies, such as modifying the regularisation term in (2) through a spatial adaptive structure, which could be more consistent with the behaviour of the deformations in medical imaging; and evaluating different alternatives for OF computation.

\section{Conclusions}

In this work, local intensity transformations were suggested to perform a multimodal NRR based on PF and iterative OF. These transformations map the target and source images into a space where the multimodal property was overcome, such that an efficient OF solution can be pursued. The evaluation of the proposal was based on CT and MR brain images deformed synthetically, and on real clinical cases. The results showed that the local entropy is the LVM with the best performance, both qualitatively and quantitatively, by analysing the anatomical structures of interest in RT (brain, tumour and ventricles). In addition, the proposal offers a solution for multimodal NRR based on quadratic optimisation over a convex surface, which allows independent motion of each pixel. This property increases the elastic deformations that could be achieved; in contrast to methods that parameterise the deformation space that limit the accuracy and increase the computational cost by non-linear optimisation processes. Therefore our NRR proposal could be considered as a new option for medical IR in RT applications by extending and evaluating the proposed approach in a $3 \mathrm{D}$ context.

\section{References}

1 Zitová, B., Flusser, J.: 'Image registration methods: a survey', Image Vis. Comput., 2003, 21, pp. 977-1000

2 Modersitzki, J.: 'Numerical methods for image registration' (Oxford University Press, New York, 2004)

3 Pluim, J.P., Fitzpatrick, J.M.: 'Image registration', IEEE Trans. Med. Imaging, 2003, 22, (11), pp. 1341-1343.

4 Nocedal, J., Wright, S.J.: 'Numerical optimization' (Springer, New York, 2006)

5 Pluim, J.P., Maintz, J.B., Viergever, M.A.: 'Image registration by maximization of combined mutual information and gradient information', IEEE Trans. Med. Imaging, 2000, 19, (8), pp. 809-814

6 Wells, W.M., Viola, P., Atsumi, H., Nakajima, S., Kikinis, R.: 'Multi-modal volume registration by maximization of mutual information', Med. Image Anal., 1996, 1, (1), pp. 35-51

7 Maes, F., Collignon, A., Vandermeulen, D., Marchal, G., Suetens, P.: 'Multimodality image registration by maximization of mutual information', IEEE Trans. Med. Imaging, 1997, 16, (2), pp. 187-198

8 Pluim, J.P., Maintz, J.B., Viergever, M.A.: 'Mutual-information-based registration of medical images: a survey', IEEE Trans. Med. Imaging, 2003, 22, (8), pp. 986-1004
9 Gonzalez, R.C., Woods, R.E., Eddins, S.L.: 'Digital image processing using MATLAB' (Gatesmark Publishing, 2009)

10 Man, K.F., Tang, K.S., Kwong, S.: 'Genetic algorithms concepts and designs' (Springer, London, 2001)

11 Arulampalam, M.S., Maskell, S., Gordon, N., Clapp, T.: 'A tutorial on particle filters for online nonlinear/non-Gaussian Bayesian tracking', IEEE Trans. Signal Process., 2002, 50, (2), pp. 174-188

12 Das, A., Bhattacharya, M.: 'Affine-based registration of CT and MR modality images of human brain using multiresolution approaches: comparative study on genetic algorithm and particle swarm optimization', Neural Comput. Appl., 2010, 20, (2), pp. 223-237

13 Arce-Santana, E.R., Campos-Delgado, D.U., Alba, A.: 'Affine image registration guided by particle filter', IET Image Process., 2012, 6, (5), pp. 455-462

14 Rueckert, D., Aljabar, P.: 'Nonrigid registration of medical images: theory, methods, and applications', IEEE Signal Process. Mag., 2010, 27, (4), pp. 113-119

15 Bin, L., Lianfang, T., Shanxing, O.: 'Rapid multimodal medical image registration and fusion in $3 \mathrm{D}$ conformal radiotherapy treatment planning'. Proc. Int. Conf. Bioinformatics and Biomedical Engineering, 2010, pp. 1-5.

16 Xuan, J., Wang, Y., Freedman, M.T., Adali, T., Shields, P.: 'Nonrigid medical image registration by finite-element deformable sheet-curve models', Int. J. Biomed. Imaging, 2006, 2006, pp. 1-9

17 Serifovic-Trbalic, A., Demirovic, D., Prljaca, N., Szekely, G., Cattin, P. C.: 'Intensity-based elastic registration incorporating anisotropic landmark errors and rotational information', Int. J. Comput. Assist. Radiol. Surgery, 2009, 4, (5), pp. 463-468

18 Klein, A., Andersson, J., Ardekani, B.A., Ashburner, J., Avants, B., et al.: 'Evaluation of 14 nonlinear deformation algorithms applied to human brain MRI registration', Neuroimage, 2009, 46, (3), pp. 786-802

19 Arce-Santana, E.R., Campos-Delgado, D.U., Alba, A.: 'A non-rigid multimodal image registration method based on particle filter and optical flow'. Proc. Sixth Int. Conf. Advances in Visual Computing, 2010 (LNCS 6453), pp. 35-44

20 Mejia-Rodriguez, A., Arce-Santana, E.R., Scalco, E., Tresoldi, D., Mendez, M.O., et al.: 'Elastic registration based on particle filter in radiotherapy images with brain deformations'. Proc. Annual Int. Conf. IEEE Engineering in Medicine and Biology Society, 2011, pp. 8049-8052

21 Reducindo, I., Arce-Santana, E.R., Campos-Delgado, D.U., Alba, A.: 'Evaluation of multimodal medical image registration based on particle filter'. Proc. Seventh Int. Conf. Electrical Engineering, Computing Science and Automatic Control, 2010, pp. 406-411

22 Reducindo, I., Arce-Santana, E.R., Campos-Delgado, D.U., Vigueras-Gomez, F.: 'Non-rigid multimodal image registration based on local variability measures and optical flow'. Proc. Annual Int. Conf. IEEE Engineering in Medicine and Biology Society, 2012, pp. $1133-1136$.

23 Simon, D.: 'Optimal state estimation' (Wiley, New Jersey, 2006)

24 Reducindo, I., Arce-Santana, E.R., Campos-Delgado, D.U., Alba, A., Vigueraz-Gomez, F.: 'An exploration of multimodal similarity metrics for parametric image registration based on particle filtering'. Proc. Eighth Int. Conf. Electrical Engineering, Computing Science and Automatic Control, 2011, pp. 1-6

25 Horn, B.K., Schunck, B.G.: 'Determining optical flow: a retrospective', Artif. Intell., 1993, 59, (1-2), pp. 81-87

26 Van Trees, H.L.: 'Detection, estimation and modulation theory: part 1' (Wiley, New Jersey, 2001)

27 Biswal, P.C.: 'Numerical analysis' (Prentice-Hall of India Pvt. Ltd., New Delhi, 2008)

28 Varian Medical Systems. 'Eclipse treatment planning systems',

2013. Available at http://www.varian.com/media/oncology/products/ eclipse/pdf/EclipseSellSheet.pdf

29 Schaefer, S., McPhail, T., Warren, J.: 'Image deformation using moving least squares'. ACM Trans. Graph., Proc. ACM SIGGRAPH 2006, 25, (3), pp. 533-540

30 McAuliffe, M.J., Lalonde, F.M., McGarry, D., Gandler, W., Csaky, K., et al.: 'Medical image processing, analysis and visualization in clinical research'. Proc. 14th IEEE Symp. on Computer-Based Medical Systems, 2001, pp. 381-386

31 Faggiano, E., Fiorino, C., Scalco, E., Broggi, S., Cattaneo, M., et al.: 'An automatic contour propagation method to follow parotid glands deformation during head-and-neck cancer tomotherapy', Phys. Med. Biol., 2011, 56, (3), pp. 775-791

32 Wang, H., Garden, A.S., Zhang, L., Wei, X., Ahamad, A., et al.: 'Performance evaluation of automatic anatomy segmentation algorithm on repeat or four-dimensional computed tomography images using 
deformable image registration method', Int. J. Radiat. Oncol. Biol. Phys., 2008, 72, (1), pp. 210-219

33 Heimann, T., Van Ginneken, B., Styner, M., Arzhaeva, Y., Aurich, V., et al.: 'Comparison and evaluation of methods for liver segmentation from CT datasets', IEEE Trans. Med. Imaging, 2009, 28, (8), pp. $1251-1265$
34 Studholme, C., Hill, D.L., Hawkes, D.J.: 'An overlap invariant entropy measure of 3d medical image alignment', Pattern Recognit., 1999, 32, (1), pp. 71-86

35 Baker, S., Scharstein, D., Lewis, J., Roth, S., Black, M., Szeliski, R.: ‘A database and evaluation methodology for optical flow', Int. J. Comput. Vis., 2011, 92, pp. 1-31 